\title{
EL IMPACTO DE LA FORMACIÓN EN COACHING ENTRE LOS DIRECTORES DE HOTEL ESPAÑOLES DE MELIÁ HOTELS INTERNATIONAL S.A.
}

Francisco M. Piedras Murillo'

Formador de Directivos de Meliá Hotels International S.A. Profesor del Máster en Dirección Hotelera de la Universidad de Sevilla pacopiedras@hotmail.com

\begin{abstract}
Resumen
El presente trabajo, mediante metodologías cuantitativa y cualitativa de investigación, confirma que los directores de los hoteles españoles de Meliá han experimentado un cambio en sus estilos directivos al que no es ajena la formación recibida sobre los principios de coaching, con estilos más participativos y mayor énfasis en el alineamiento de esfuerzos y la escucha activa de sus colaboradores, actitudes compatibles con la máxima responsabilidad del directivo: fijar prioridades. La aplicación de dichos principios al trabajo diario suscita un amplio consenso - mayor entre los jóvenes que entre los veteranos- por lo que reclaman su inclusión en la formación de ejecutivos hoteleros.
\end{abstract}

\section{Palabras clave}

alineamiento, directivo-coach, directores, hotel, escucha activa, estilos directivos, formación, meliá

\begin{abstract}
Through both quantitative and qualitative methods of research, this article confirms that the general managers of the Spanish Meliá hotels have seen a change in their management style, being one of the main reasons the training received in coaching principles. This change has led to more participatory styles, a deeper alignment of efforts within departments and a greater inclination to listen to employees, attitudes compatible with the highest responsibility of the management team: to set priorities. The application of these principles in the day-to-day routine raises a broad consensus, deeper, however, amongst younger managers than veterans. Consequently, they opt to include them in the training of hotel executives.
\end{abstract}

\section{Keywords}

alignment, manager as a coach, general managers, hotel, active listening, managerial styles, training, melia

\section{INTRODUCCIÓN}

Aunque el presente trabajo se limite a los directores de sus hoteles españoles, Meliá Hotels Internacional S.A. es una compañía de clara dimensión internacional. Con más de 350 hoteles en 35 países y 4 continentes, declara como misión "ofrecer experiencias y servicios globales de alojamiento con criterios de excelencia, responsabilidad y sostenibilidad".

De entre las tareas de sus directores cabe destacar la de trabajar de manera incansable para que todos los departamentos funcionen como un todo armónico. "Si tuviera que pronunciarme sobre la función fundamental por la que un director de hotel se gana su sueldo, sin duda me inclinaría por lograr el alineamiento de esfuerzos de todos los departamentos... Sus jefes siempre la negarán [la confrontación entre departamentos] pero bajo una fuerte presión... los roces y descoordinaciones se repiten hasta el infinito. Por ello, tener que recordar una y mil veces los mismos mensajes de alineamiento a los componentes del equipo se configura como una tarea ingrata y agotadora para el director porque... nadie parece recordarlos cuando pintan bastos" (Piedras, $2008: 31$ 33). Conceptos tales como Coaching, Liderazgo Situacional y flexibilidad adquieren carta de naturaleza en la consecución de ese necesario alineamiento de los recursos hoteleros.

\section{MARCO TEÓRICO}

\subsection{Breve aproximación al Coaching}

De las múltiples definiciones de coaching escogeremos solo algunas, muy clarificadoras. El coaching "apunta a mejorar el desempeño y la capacidad de aprendizaje de los demás. Incluye proporcionar feedback... motivación, preguntas eficaces y ajustar de forma consciente el propio estilo directivo a la predisposición del coachee para realizar una determinada tarea" (Landsberg, 1997: xi). Significa "crear la atmósfera ideal para el éxito del empleado a través de una continua comunicación, escucha activa de sus necesidades, reconocimiento de

\footnotetext{
${ }^{1}$ Doctor en Turismo por la Universidad de Sevilla, Facultad de Turismo y Finanzas.
} 
buenos desempeños, reorientación de desempeños incorrectos y apoyo continuo para desempeños futuros" (Crinelli y Maigret, 1999: 22) y recomienda un paso atrás del directivo para ceder protagonismo a quienes, en definitiva, realizan las tareas.

Las técnicas de coaching reafirman también el derecho de la persona a tomar decisiones con plena libertad y afrontar sus consecuencias. Los existencialistas -"el Coaching retoma de la filosofía existencialista... la toma de conciencia de los propios recursos y limitaciones” (Martínez González, 2011: 4)- ya lo advertían en el siglo pasado: tenemos que elegir por nuestra cuenta cómo queremos vivir...; somos individuos libres, y debido a nuestra libertad estamos condenados a elegir durante toda la vida; las elecciones que hacemos resultan muy importantes porque somos completamente responsables de todos nuestros actos (Sartre, 1949). Son, por tanto, las acciones -y no las intenciones- que decidimos tomar las que delimitan nuestras responsabilidades: "somos lo que hacemos, no lo que decimos o pensamos" (Crinelli, 1999: 21). Ya lo postuló, mucho antes, Aristóteles: somos lo que repetidamente hacemos.

\subsection{Tipos de Coaching}

El coaching se sustancia como un proceso de acompañamiento y apoyo al directivo con tres posibilidades: un coach externo perteneciente a un gabinete de consultoría, un coach interno perteneciente a la misma estructura pero diferente servicio, o el directivo coach, que es al mismo tiempo el responsable jerárquico y el coach de los miembros clave de su equipo (Gautier y Vervisch, 1997). Es precisamente en el papel de directivo coach en el que va a centrarse el presente trabajo sobre las consecuencias prácticas de acciones formativas directas a directivos hoteleros, tanto como factor coadyuvante de la evolución de sus estilos directivos como en la inclusión que demandan de los principios de coaching en la formación reglada de gestión hotelera.

Los procesos formales de coaching pueden hacer que el directivo adquiera más conciencia y asuma un rol más flexible, siendo más autoritario o, por el contrario, delegando más según las situaciones y circunstancias, más allá de sus propios gustos, rasgos y preferencias (Bayón, 2010 y Jericó, 2002). Es la base del Liderazgo Situacional (Hersey, 1985), por el que el líder adapta voluntariamente su estilo directivo a los perfiles -actitud y aptitud- de sus colaboradores. Otros autores defienden que, con independencia de los procesos formales, el directivo formado en tales técnicas -o líder coach- asume con sus colaboradores un rol muy próximo al del profesional externo del coaching ejecutivo, lo que le distinguiría del gerente tradicional (Giménez, 2005 y Cuadrado, 2006).

\subsection{Cursos "el proceso de Coaching"}

Con el objeto de potenciar el liderazgo de sus ejecutivos hoteleros, desde 1999 se han venido impartiendo en Meliá H.I. cursos presenciales de dieciséis horas lectivas en los que se desarrollan y practican los principios de "El proceso de Coaching" (Crinelli y Maigret, 1999) impartidos por sus autores a los directores de hoteles españoles en 1999 y 2000 y, desde entonces, por el formador corporativo tras obtener la expresa autorización de aquellos. Estos principios son, entre otros, los siguientes:

- El directivo es el jefe pero solo el jefe: sobre él recae toda la responsabilidad pero no tiene todas las respuestas.

- Al directivo le interesa lo que piensan sus colaboradores y les pide opiniones y propuestas en las áreas de su competencia.

- El coaching como herramienta de alineamiento. Un establecimiento hotelero necesita del esfuerzo constante del directivo por conseguir que sus departamentos colaboren en pos del objetivo común.

- El impacto del coaching en el desempeño y desarrollo personales de los colaboradores. De forma prospectiva en la fijación de objetivos y tareas, adecuando los estilos a los perfiles de los colaboradores según postula el Liderazgo Situacional (Hersey y Blanchard, 1969), y evitando la frustración creada por el Management Mismatch (Crinelli y Maigret, 1999) o la inadecuación de tareas (Csikszentmihalyi, 1996 y Cuadrado, 2006). De forma retrospectiva, en los seguimientos puntuales o sistemáticos de tareas y desempeño, impregnando unos planes de acción motivadores y, en su caso, reorientadores.

- «Coaching no significa dirección blandita». Fórmula coloquial de postular que la aplicación de estos principios no conlleva una actitud de sometimiento a los colaboradores sino que enfatiza la irrenunciable responsabilidad del directivo de conseguir los objetivos empresariales: "la gestión actual es participativa pero no democrática, que no es lo mismo... En dirección, si no controlas, descontrolas, no hay término medio" (Muro, 2003: 167 y 173).

En total han sido 49 los cursos sobre coaching impartidos en tres idiomas y ocho países a 766 directivos de Meliá H.I. desde 1999 a 2011 con una aceptación global de un 90,92\% y un grado de aplicación práctica de los contenidos al trabajo diario, según los asistentes, de un 93,34\%.

\section{METODOLOGÍA}

El presente trabajo confirma que los principios de coaching recogidos en el capítulo anterior son aplicados en la práctica -si bien con ciertos matices en función de su antigüedad en el puesto- por el colectivo objeto de 
estudio, provocando cambios positivos en sus relaciones con los colaboradores y particularmente en sus estilos de dirección: en consecuencia, piden su inclusión en la formación reglada de futuros ejecutivos hoteleros.

La necesidad de objetivizar en lo posible sus opiniones en torno a estos efectos aconseja un enfoque cuantitativo mediante la utilización del cuestionario adecuado. Sin embargo, hay matices difícilmente detectables con tal metodología, por lo que se ha complementado con un enfoque cualitativo de entrevistas personales directas. Son múltiples los autores que no ven incompatibilidad alguna entre ambos enfoques y apuestan por usarlos de forma conjunta: "la utilización de varios métodos se puede realizar simultanea o secuencialmente, respetando en todo momento el carácter específico de cada método y no provocando la mezcla y el desorden" (Rodríguez et al, 1996: 69); "cabe la posibilidad de utilizar métodos cualitativos que puedan dar respuesta a algunos aspectos concretos de la investigación" (Morse, 2005: 25).

\section{1. Censo de directores y segmentación}

Meliá H.I. cedió el censo de directores y subdirectores de sus hoteles españoles, incluyendo a los recientemente jubilados y tomando como referencia la última oleada sistemática de cursos de coaching en España. Ambos listados se recibieron en mayo de 2010 y se distribuyeron en los siguientes grupos:

I. Jubilados con posterioridad a 2007: 7 personas.

II. Nacidos antes de 1952, próximos a la edad de jubilación: 22.

III. $\quad$ Con menos de cinco años de antigüedad en el puesto: 20.

IV. $\quad$ Directores consolidados, no comprendidos en los grupos II y III anteriores: 68.

V. Directores residentes y subdirectores con proyección directiva: 23.

\section{2. Enfoque cuantitativo: cuestionario y prueba de significación estadística.}

Del cuestionario general remitido a los integrantes del censo se han seleccionado las preguntas relacionadas con este trabajo: los principios de coaching del epígrafe 2.3 anterior, su aplicación práctica real y la conveniencia de incluirlos en la formación de los futuros directores de hotel. Son las siguientes:

C.1) Solo soy el jefe: no tengo todas las respuestas. En mi equipo puede haber quien haga las cosas mejor que yo.

C.2) En temas de su competencia, debo pedir opinión a mis colaboradores y profundizar en sus respuestas con las herramientas "Por qué" y "Para qué".

C.3) Debo escoger mis estilos directivos en función de los perfiles individuales de mis colaboradores directos, y no emplear siempre el que me sea más afín.

C.4) En situaciones de conflicto con mis colaboradores, debo esforzarme en buscar zonas de interés común Empresa/Directivo/Colaborador.

C.5) Soy libre para ejercer mis elecciones y, como consecuencia, debo aceptar sus consecuencias; somos lo que hacemos, no lo que decimos o pensamos.

C.6) Coaching no significa dirección débil: los objetivos que me son encomendados deben ser siempre prioritarios.

C.7) Como directivo hotelero, ¿qué utilidad práctica concedes en su conjunto a la formación en coaching recibida? -Control de consistencia para las respuestas a las seis cuestiones anteriores-.

B.7) En mi opinión, deben incorporarse a los programas formativos oficiales de Dirección Hotelera las siguientes habilidades directivas: B7a) coaching...

Los participantes debían valorar de uno a diez la importancia concedida a las primeras siete afirmaciones, significando 1 "nada importante" y 10 "importantísimo" 2 . Sin embargo, el grado de acuerdo con la cuestión B7a) se midió con una escala Likert de cinco niveles, desde "totalmente en desacuerdo" -valor 1- a "totalmente de acuerdo" -valor 5-.

El cuestionario fue remitido por correo electrónico de forma personalizada a comienzos de junio de 2010 a todos los integrantes del censo, previéndose una respuesta superior al $80 \%$. Para conocer no solo los resultados de la encuesta sino también el grado de homogeneidad de las respuestas, se realizó un análisis no paramétrico de significación estadística, en este caso la prueba de Kruskal-Wallis de muestras independientes (Canavos, 1988), por la que solo adquieren significación estadística las diferencias en las respuestas de los distintos grupos si pvalor es menor que 0,05 y no en caso contrario.

\section{3. Enfoque cualitativo: selección y entrevistas.}

La investigación de los fenómenos turísticos es terreno abonado para la aplicación de criterios cualitativos compatibles con el enfoque cuantitativo. La fuente primordial de información han sido entrevistas realizadas con las siguientes recomendaciones: selección de informantes clave que ocupen puestos importantes o cercanos a las tomas de decisiones, que puedan ofrecer visiones diferentes y contrastadas del fenómeno a estudiar, y que

\footnotetext{
${ }^{2}$ A precisar que las escalas intentan objetivar percepciones personales por lo que en realidad se trata de un análisis cuantitativo realizado sobre variables cualitativas.
} 
hablen abierta y libremente; en sus puestos de trabajo y grabadas digitalmente... (Castellanos-Verdugo et al, 2010). El amplio conocimiento que tiene el autor de los directores y subdirectores de Meliá H.I. proporciona un poderoso argumento para las entrevistas: en efecto, "una característica estratégica importante para este trabajo se refiere al papel del investigador en su trato -intensivo- con las personas involucradas en el proceso de investigación, para entenderlas" (Mendoza, 2006: 2). En consecuencia, se han seguido los siguientes pasos:

1. Selección de seis informantes entre los integrantes del censo -forzosamente, en número restringido (Yin, 1993) - repartidos, proporcionalmente a su tamaño, entre los cuatro grupos de directivos activos.

Tabla 3.1: Distribución de entrevistas por grupos

\begin{tabular}{|c|c|c|c|}
\hline \multicolumn{4}{|c|}{ CENSO DH ESPANA MELIA H.I. } \\
\hline GRUPO & Depurado & $\%$ & Entrevistas \\
\hline I & 7 & 5,00 & 0 \\
\hline II & 22 & 15,71 & 1 \\
\hline III & 20 & 14,29 & 1 \\
\hline IV & 68 & 48,57 & 3 \\
\hline V & 23 & 16,43 & 1 \\
\hline Totales & 140 & 100,00 & 6 \\
\hline
\end{tabular}

Fuente: Elaboración propia

Numerosa literatura respalda la elección no aleatoria de los directivos a entrevistar (Fraenkel y Wallen, 1996 o Quintana y Montgomery, 2006). Véase en la tabla 3.2 siguiente el criterio objetivo escogido: la menor distancia de las respuestas de los encuestados seleccionados a los promedios de sus respectivos grupos.

Tabla 3.2: Directores a entrevistar

\begin{tabular}{|c|c|c|c|c|}
\hline \multicolumn{5}{|c|}{ DIRECTORES A ENTREVISTAR } \\
\hline GRUPO & $\mathbf{N}^{\circ}$ Censo & Distancia a la media & Media grupo & $\%$ coincidencia \\
\hline II & 104 & 1,61 & 55,37 & 97,09 \\
\hline III & 67 & 1,72 & 58,42 & 97,06 \\
\hline IV & 85 & 1,63 & 55,22 & 97,05 \\
\hline IV & 24 & 1,70 & 55,22 & 96,92 \\
\hline IV & 42 & 1,75 & 55,22 & 96,83 \\
\hline V & 115 & 1,53 & 59,85 & 97,44 \\
\hline
\end{tabular}

Fuente: Elaboración propia

2. Confección del guión para las entrevistas que incluye datos del hotel, circunstancias personales y visión de su trabajo, descripción de un día tipo, situaciones difíciles con resultado de éxito o fracaso -al modo de las "Behavioural Event Interviews desarrolladas por McLelland y Dayley en 1972" (Dalziel et al, 1996: 27)formación recibida, opinión sobre los principios de coaching y su impacto en su forma de dirigir y materias que incluiría en los programas de formación de directivos hoteleros. Para contrastar las opiniones de cada director se ha entrevistado también a un jefe de departamento cercano a él y con una antigüedad mínima de cinco años, garantizando así su solvencia profesional y un estrecho contacto con su superior. Para ellos, las preguntas se centran en su día a día, en la relación con sus distintos directores y en las diferencias entre sus formas de dirigir. Naturalmente no se descarta ahondar durante las mismas en otros aspectos no planificados si interesa a la investigación (Quintana y Montgomery, 2006).

3. Realización de las entrevistas entre 2010 y 2011 en sus propios hoteles, ubicados en Andalucía, Baleares, Canarias, Cataluña y Valencia. Se ha garantizado el anonimato de los entrevistados utilizando claves - "D" para directivos, "J" para jefes y "H" para los hoteles seguidos del numeral del grupo- y adoptando el género masculino, mayoritario, en todos los análisis.

Tabla 3.3: Entrevistas y claves para el análisis

\begin{tabular}{|c|c|c|c|c|}
\hline GRUPO & N $^{\circ}$ Censo & Posición & Clave & Hotel \\
\hline Entrevista de prueba & Director & D0 & H0 \\
\hline II & 104 & Director & D2 & H2 \\
\hline II & s/n & J. Admon. & J2 & H2 \\
\hline III & 67 & Director & D3 & H3 \\
\hline III & s/n & J. Recepción & J3 & H3 \\
\hline IV & 85 & Director & D4A & H4A \\
\hline IV & s/n & J. Calidad & J4A & H4A \\
\hline IV & 24 & Director & D4B & H4B \\
\hline IV & s/n & J. Recepción & J4B & H4B \\
\hline IV & 42 & Director & D4C & H4C \\
\hline IV & $\mathbf{s} / \mathbf{n}$ & J. Recepción & J4C & H4C \\
\hline V & 115 & Subdirector & D5 & H5 \\
\hline V & $\mathbf{s} / \mathbf{n}$ & J.Recepción & J5 & H5 \\
\hline
\end{tabular}

Fuente: Elaboración propia 
4. Grabación y posterior trascripción y archivo en soporte informático. Para procurar la familiarización del autor con el guión y la técnica de las entrevistas, se realizó una prueba en un hotel de 4 estrellas andaluz ajeno a Meliá H.I., por lo que su director -D0- no está incluido en el censo ni su análisis individual recogido en las conclusiones.

\section{RESULTADOS DEL ENFOQUE CUANTITATIVO}

Véase en la tabla 4.1 siguiente que prácticamente todos los cuestionarios remitidos fueron cumplimentados y devueltos dentro del plazo convenido, siendo inferior al $3 \%$ el porcentaje de los que no contestaron.

Tabla 4.1: Nivel de respuesta al cuestionario

\begin{tabular}{|c|c|c|c|}
\hline \multicolumn{5}{|c|}{ RESPUESTAS OBTENIDAS } \\
\hline GRUPO & Respuestas & Censo neto & $\%$ s/censo \\
\hline I & 5 & 7 & 71,43 \\
\hline II & 22 & 22 & 100,00 \\
\hline III & 20 & 20 & 100,00 \\
\hline IV & 66 & 68 & 97,06 \\
\hline V & 23 & 23 & 100,00 \\
\hline Totales & 136 & 140 & 97,14 \\
\hline
\end{tabular}

Fuente: Elaboración propia

\subsection{Análisis descriptivo de las respuestas}

Tabla 4.2: Tabulación de respuestas al apartado c) de coaching

\begin{tabular}{|c|c|c|c|c|c|c|c|c|c|c|c|c|c|c|c|c|c|c|}
\hline \multirow{2}{*}{ Items } & \multicolumn{3}{|c|}{ Grupo I } & \multicolumn{3}{|c|}{ Grupo II } & \multicolumn{3}{|c|}{ Grupo III } & \multicolumn{3}{|c|}{ Grupo IV } & \multicolumn{3}{|c|}{ Grupo V } & \multicolumn{3}{|c|}{ TOTAL } \\
\hline & Freq. & MED & MOD & Freq. & MED & MOD & Freq. & MED & MOD & Freq. & MED & MOD & Freq. & MED & MOD & Freq. & MED & MOD \\
\hline C1 & 4 & 8,50 & 10,00 & 19 & 8,50 & 8,00 & 19 & 9,42 & 10,00 & 58 & 8,72 & 8,00 & 20 & 9,70 & 10,00 & 120 & 8,95 & 10,00 \\
\hline $\mathrm{C2}$ & 4 & 8,25 & 8,00 & 19 & 8,83 & 10,00 & 19 & 9,53 & 10,00 & 58 & 8,97 & 10,00 & 20 & 9,50 & 10,00 & 120 & 9,10 & 10,00 \\
\hline C3 & 4 & 7,75 & 8,00 & 19 & 8,28 & 9,00 & 19 & 9,37 & 10,00 & 58 & 8,76 & 8,00 & 20 & 9,25 & 10,00 & 120 & 8,83 & 10,00 \\
\hline $\mathrm{C} 4$ & 4 & 9,25 & 9,00 & 19 & 9,11 & 10,00 & 19 & 9,16 & 10,00 & 58 & 8,90 & 10,00 & 20 & 9,15 & 10,00 & 120 & 9,03 & 10,00 \\
\hline C5 & 4 & 8,25 & N.A. & 19 & 8,50 & 9,00 & 19 & 9,05 & 10,00 & 58 & 8,57 & 10,00 & 20 & 8,80 & 10,00 & 120 & 8,66 & 10,00 \\
\hline C6 & 4 & 9,00 & 10,00 & 19 & 9,06 & 9,00 & 19 & 9,11 & 10,00 & 58 & 9,02 & 10,00 & 20 & 9,35 & 9,00 & 120 & 9,09 & 10,00 \\
\hline C7 & 4 & 9,50 & 10,00 & 19 & 9,33 & 10,00 & 19 & 9,53 & 10,00 & 58 & 9,03 & 10,00 & 20 & 9,45 & 10,00 & 120 & 9,24 & 10,00 \\
\hline
\end{tabular}

(Leyendas: Freq.: frecuencia absoluta; MED: media aritmética; MOD: moda.)

Fuente: Elaboración propia ${ }^{3}$

Respecto de las preguntas C.1) a C.7), valoradas de 1 a 10 -recuérdese que las primeras seis corresponden a los principios clave de coaching y la séptima a su aplicación práctica general-, destaca que la moda u opción más elegida fue 10 para cada uno de los items. Las medias de las respuestas a las primeras seis preguntas oscilaron desde 8,66 para el item C.5) hasta 9,10 para el C.2), siendo el promedio más alto, 9,24, precisamente para la pregunta de control, C.7). Puede, pues, calificarse de prácticamente unánime el respaldo de los encuestados a la relevancia de los principios de coaching para su trabajo diario. Profundizando el análisis, se identifican dos escalones bien definidos: un primer nivel en torno a 9,3 en los promedios de los grupos III y V, y un segundo nivel de 8,8 en los grupos II y IV, lo que sugiere, dentro de la sobresaliente aceptación general de los principios, una mayor naturalidad en su asimilación por parte de los directivos noveles que por los ya consolidados.

Respecto de la afirmación B.7a) sobre que los principios de coaching debieran incluirse en los programas formativos oficiales de dirección hotelera, el grado de acuerdo entre los participantes alcanzó, en la escala Likert de cinco niveles, un promedio de 4,87 -un 97\%- en una estrecha horquilla desde 4,50 del grupo de jubilados hasta 4,95 de los grupos de veteranos y noveles; el grupo mayoritario de directores consolidados obtuvo una media de 4,86, siendo 5 la moda de todos los grupos, de nuevo su valor máximo posible. No cabe, pues, duda alguna sobre el posicionamiento favorable de los directores hacia la cuestión planteada.

\subsection{Análisis inferencial de las respuestas}

El análisis no paramétrico de las opiniones sobre la aplicación al trabajo diario de los principios de coaching mediante la prueba de Kruskal-Wallis de muestras independientes proporciona la necesaria consistencia estadística a las conclusiones del análisis descriptivo anterior; véanse sus resultados en la tabla 4.3 siguiente y recuérdese que para que las diferencias entre las respuestas de los distintos grupos alcancen significación estadística, la columna "p-valor" no debe sobrepasar el valor de referencia 0,05.

\footnotetext{
${ }^{3}$ La diferencia entre las respuestas totales y las tabuladas corresponde a los 16 cuestionarios depurados por respuestas incoherentes a la pregunta general de control C.7) en relación con el resto de preguntas C.1) a C.6).
} 
Tabla 4.3: Significación estadística de las diferencias entre grupos de las respuestas a las preguntas c. 1 a $c .7$ y b.7a

\begin{tabular}{|c|c|l|c|}
\hline Ord & Item & \multicolumn{1}{|c|}{ Descripción } & p-valor \\
\hline $1^{\circ}$ & C.1 & Solo soy el jefe y no tengo todas las respuestas & 0,001 \\
\hline $2^{\circ}$ & C.2 & $\begin{array}{l}\text { Escuchar siempre a los colaboradores en los temas de } \\
\text { su competencia }\end{array}$ & 0,025 \\
\hline $3^{\circ}$ & C.3 & $\begin{array}{l}\text { Escoger los estilos directivos en función de los perfiles } \\
\text { individuales de los colaboradores }\end{array}$ & 0,031 \\
\hline \hline $4^{\circ}$ & B.7a & $\begin{array}{l}\text { Deben incorporarse las técnicas de coaching a los } \\
\text { programas formativos oficiales de Dirección Hotelera }\end{array}$ & 0,087 \\
\hline $5^{\circ}$ & C.7 & $\begin{array}{l}\text { Utilidad práctica del conjunto de la formación en } \\
\text { Coaching }\end{array}$ & 0,366 \\
\hline $6^{\circ}$ & C.5 & $\begin{array}{l}\text { Soy libre para ejercer mis elecciones y debo aceptar } \\
\text { sus consecuencias }\end{array}$ & 0,415 \\
\hline $7^{0}$ & C.6 & $\begin{array}{l}\text { Coaching no significa dirección débil: los objetivos } \\
\text { deben ser prioritarios. }\end{array}$ & 0,822 \\
\hline $8^{0}$ & C.4 & $\begin{array}{l}\text { En situaciones de conflicto, debo buscar las zonas de } \\
\text { interés común. }\end{array}$ & 0,877 \\
\hline
\end{tabular}

(Leyenda: Ord.: ordinal)

Fuente: Elaboración propia (prueba de Kruskal-Wallis)

En consecuencia, no existen diferencias significativas entre las respuestas de los distintos grupos a las preguntas de C.4) a C.7) y B.7a) pero sí en las de las preguntas C.1) a C.3). Con independencia del alto grado de coincidencia de los encuestados con los principios de coaching, parecen confirmarse las reticencias de algunos grupos -ya apuntadas en el análisis descriptivo anterior- a la hora de aceptar los tres primeros, "solo soy el jefe", "debo escuchar siempre a mis colaboradores" y "debo escoger mis estilos directivos en función de los perfiles de los colaboradores", lo que sugiere en ellos cierto sustrato autoritario.

Puede, por consiguiente, afirmarse que tanto el análisis descriptivo del epígrafe 4.1 anterior como la presente prueba de significación estadística avalan la utilidad práctica de la formación en los principios de coaching para el trabajo diario de los directores de hotel de Meliá H.I., reclamando su inclusión en los planes de estudio de futuros directivos hoteleros. Asimismo, y dentro de una sobresaliente aceptación general de tales principios, se observan ciertos matices diferenciadores entre los grupos, a confirmar por el enfoque cualitativo posterior.

\section{RESULTADOS DEL ENFOQUE CUALITATIVO: ANÁLISIS DE CASOS MÚLTIPLES}

La necesaria limitación de espacio impide recoger aquí los análisis individuales de las entrevistas. Por lo tanto Incluiremos solo el análisis de casos múltiples basado en aquellos, si bien se incluye tras el epígrafe 5.3 un resumen de todas las entrevistas como tabla 5.1.

\subsection{Análisis de los estilos directivos}

El primer análisis corresponde a los tres directores del grupo IV, que suponen la mitad de las entrevistas realizadas y representan al $48,57 \%$ del censo. De sus casos individuales se desprende una considerable coincidencia de planteamientos. Sesgados por su propia e interesada visión, sus estilos directivos aparecen como formalmente participativos aunque con cierto sustrato coercitivo, no siempre reconocido: "flexible", "no estoy encima", "dejo trabajar", "delego" son sus propias expresiones, pero también "sé cómo lo quiero" o "todo bajo control". Sus colaboradores directos coinciden con ellos: "escuchan", "son accesibles" y "explican", pero "deciden". Y si bien escuchan, aceptan lo que proponen sus colaboradores "unas veces sí y otras no". Hay claras trazas de este sustrato coercitivo en dos de los integrantes del grupo y algo menos en D4B, cuya ausencia de auto-crítica y su displicente frase "a los buenos jefes se les puede dejar trabajar" apuntan también en la misma dirección.

Entre los representantes de los directores noveles y subdirectores las similitudes son asimismo numerosas. Ambos se muestran -y sus colaboradores directos lo corroboran- dialogantes, cercanos, participativos y suaves en las formas. Sin embargo, mientras el director novel practica una delegación condicionada y controla tanto los objetivos como la forma de conseguirlos, el subdirector concede a sus colaboradores una autonomía real, controlando solo las metas y dejándoles decidir sobre cómo conseguirlas. A pesar de ello, sus respectivos jefes vinculados reconocen a éste "firmeza cuando corresponde" mientras achacan a aquel director "cierta debilidad disciplinaria", lo que desmiente el aparente binomio inexperiencia-falta de carácter. Por último, el representante del grupo de directores próximos a la jubilación destaca por su estilo paternalista y orientador: en él, y a diferencia de los del resto de los grupos, los rasgos autoritarios se evidencian con claridad.

\subsection{Aplicación práctica de los principios de coaching}

La influencia de los principios de coaching, aunque unánimemente reconocida, arroja ciertos matices entre los tres directores consolidados. D4A los considera de aplicación práctica indudable y esencial para ajustar los 
estilos directivos a las circunstancias; D4B va más lejos y afirma tener "siempre sobre la mesa una carpeta con las herramientas [de coaching] para recordar utilizarlas en el día a día" y las considera incluso más importantes que los conocimientos operativos; y D4C declara dedicarles nada menos que el $70 \%$ de su tiempo porque "le ayudan a llegar a la gente" y porque su aplicación le resulta muy útil tanto a nivel personal como profesional. Coincidencia notable, pues, entre quienes ya atesoran una importante experiencia.

El resto del análisis extrae parecidas similitudes entre los representantes de los grupos de directores noveles y subdirectores: mientras el primero enfatiza su utilidad para motivar a los colaboradores y a practicar correctamente la delegación de tareas, el segundo destaca su "aplicación positiva diaria y constante" que afecta a la escucha activa, al clima organizativo y a la toma de decisiones. Sin embargo, el representante del grupo de veteranos se muestra escéptico al respecto y, aunque afirma que los principios de coaching influyen en su estilo directivo -quizá para no salirse del guión-, no concreta ninguno y defiende que "su aplicación depende de las circunstancias". Tal actitud contrasta con la alta aceptación media de los principios de este grupo II en los cuestionarios -9,33 sobre 10- pero se alinea con algunas de sus reticencias hacia los que suponen una mayor cesión de protagonismo: "no tengo todas las respuestas", "soy libre para ejercer mis decisiones" y, sobre todo, "debo adaptar mis estilos directivos a los perfiles de mis colaboradores".

Puede pues ratificarse la conclusión del análisis cuantitativo anterior en cuanto a que los principios de coaching son de utilidad práctica en el trabajo diario del colectivo estudiado. Además, la salvedad del último párrafo aporta verosimilitud a que tales principios son aceptados con mayor naturalidad por los directores jóvenes que por los ya consolidados.

\subsection{Inclusión de habilidades directivas en la formación hotelera}

La formación en habilidades directivas fue echada de menos por los directores consolidados cuando cursaron sus estudios reglados; los tres, como ya se ha recogido en el primer párrafo del epígrafe anterior, destacan el coaching como una de las más importantes "tanto a nivel personal como profesional" y demandan su inclusión en la formación directiva hotelera. A esta petición se suman los representantes de los directores noveles y subdirectores, precisando además otras habilidades complementarias como comunicación y técnicas de negociación. El representante de los directores veteranos reclama una "formación en Psicología aplicada a los recursos humanos", lo que, en el fondo, coincide con el resto de los grupos. No debe sorprender tal unanimidad en un colectivo muy homogéneo al que su empresa ha facilitado amplia y sistemática formación sobre las habilidades directivas citadas, amén de monitorizar estrechamente el clima organizativo de sus unidades.

Tabla 5.1: Resumen de las entrevistas a directores

\begin{tabular}{|c|c|c|}
\hline DH & Coaching en la práctica & Formación en habilidades \\
\hline D2 & $\begin{array}{c}\text { Escéptico en el fondo. Su aplicación } \\
\text { depende de las circunstancias. Afecta a su } \\
\text { estilo directivo. Solo recuerda anécdotas. }\end{array}$ & $\begin{array}{c}\text { Falta formación en Psicología aplicada a los } \\
\text { recursos humanos. }\end{array}$ \\
\hline D3 & $\begin{array}{c}\text { Sobre todo en el trato con los } \\
\text { colaboradores. Ayuda a delegar y motivar. } \\
\text { Útil para mandos medios. }\end{array}$ & $\begin{array}{c}\text { Faltan habilidades directivas en las escuelas: } \\
\text { Liderazgo, Comunicación y Negociación. }\end{array}$ \\
\hline D4A & $\begin{array}{c}\text { De aplicación indudable. Según ubicación } \\
\text { geográfica. Esencial para ajustar estilos a } \\
\text { perfiles y circunstancias. }\end{array}$ & $\begin{array}{c}\text { TEAT: sin habilidades directivas. } \\
\text { Posterior formación sobre habilidades en la } \\
\text { empresa. Cambiaron su estilo directivo. }\end{array}$ \\
\hline D4B & $\begin{array}{c}\text { Mantiene los principios del Coaching sobre } \\
\text { su mesa. Más importantes aún que los } \\
\text { conocimientos operativos. }\end{array}$ & $\begin{array}{c}\text { Las echó de menos en la Diplomatura. } \\
\text { Posterior formación en la empresa. } \\
\text { Coaching sobre todo. }\end{array}$ \\
\hline D4C & $\begin{array}{c}\text { Útil tanto a nivel personal como profesional. } \\
\text { Lo usa el 70\% de su tiempo. } \\
\text { Ayuda a llegar a la gente. }\end{array}$ & $\begin{array}{c}\text { Solo técnica en Diplomatura y Máster. } \\
\text { Formación posterior en empresa (Coaching, OW, } \\
\text { Trypducción.). }\end{array}$ \\
\hline D5 & $\begin{array}{c}\text { Aplicación positiva diaria y constante. } \\
\text { Afecta a la escucha, al clima y a la toma de } \\
\text { decisiones. }\end{array}$ & $\begin{array}{c}\text { Solo formación técnica en la Diplomatura. } \\
\text { Formación posterior en la empresa: Coaching, } \\
\text { negociaciones, presentaciones. }\end{array}$ \\
\hline \hline
\end{tabular}

(Leyenda: DH: clave del director de hotel)

Fuente: Elaboración propia

\section{CONCLUSIONES}

Los principios de coaching son de aplicación práctica en el trabajo diario de los directores de hotel españoles de Meliá H.I., afirmación respaldada: a) por la investigación cuantitativa, merced al análisis descriptivo del epígrafe 4.1 y la prueba Kruskal-Wallis para muestras independientes del 4.2, y b) por la investigación cualitativa mediante el análisis múltiple de las entrevistas del 5.2. Dentro de su elevada aceptación general, no existen diferencias significativas respecto de los tres principios "búsqueda de zonas de interés común", "soy libre para ejercer mis elecciones" y "coaching no significa dirección débil". En cambio, sí las hay respecto de los que suponen cierta cesión de protagonismo -"solo soy el jefe", "escuchar siempre a los colaboradores" y "escoger los estilos directivos en función de los perfiles de éstos"-, principios que despiertan reticencias en algunos directores, particularmente los de mayor edad. La investigación cualitativa posterior también abona con firmeza 
la más natural aceptación de los principios de coaching por los subdirectores y directores noveles que por los veteranos ya consolidados. Por último, los directores de hoteles españoles de Meliá H.I. demandan la inclusión, en la formación reglada de directivos hoteleros, de tales principios como habilidad directiva, afirmación respaldada por el análisis descriptivo del epígrafe 4.1, la prueba Kruskal-Wallis para muestras independientes del 4.2 y el análisis cualitativo del 5.3. El colectivo objeto del presente trabajo observa serias carencias en la mayoría de alumnos en prácticas, consecuencia de los inadecuados planes de estudio tradicionales, denunciados, entre otros, por la CEOE (areaRH.com, 2002) o la Asociación Española de Directores de Hotel (AEDH, 1997) y aún no completamente superados.

\section{REFERENCIAS BIBLIOGRÁFICAS}

AEDH, Asociación Española de Directores de Hotel (1997): Comunicado final de la 25a Asamblea General, Benalmádena, Málaga.

AreaRH (2002): "La movilidad funcional llega a la empresa". Consultado el 19.10.2011 en http://www.arearh.com/actualidad/2002/0521htlm

Bayón, F. (2010): "Habilidades directivas, coaching". Calidad (3), 28-31.

Canavos, G.C. (1988): Probabilidad y Estadística, Aplicaciones y métodos, McGraw-Hill Interamericana de México, México.

Castellanos-Verdugo, M., Caro, F. y Oviedo-Garcia, M.A. (2010): "An Application of Grounded Theory to Cultural Tourism Research: Resident Attitudes to Tourism Activity in Santiponce". Cultural Tourism Research Methods. Oxfordshire, Reino Unido. Cabi Publishing.

Crinelli, P. y Maigret, O. (1999): "Le processus de Coaching", curso para directores de hotel de Meliá H.I., Palma de Mallorca.

Crinelli, P. (1999) : Réussir au pluriel - Agir en interdépendence, Éditions d'Organisation, Paris.

Csikszentmihalyi, M. (1996): FLUIR. Una psicología de la felicidad. Kairós. Barcelona.

Cuadrado, D. (2006). "Cuando el líder hace crecer (I)". Capital Humano (19)198, 34-44.

Dalziel, M., Cubeiro, J.C. y Fernández, G., HayGroup (1996): Las competencias: clave para una gestión integrada de los Recursos Humanos. Deusto. Bilbao.

Fraenkel y Wallen (1996): Investigación cualitativa, citado por Vera, L., UIPR, Ponce, P.R. Consultado en http://ponce.inter.edu/cai/reserva/lvera/INVESTIGACION_CUALITATIVA.pdf el 05.05. 2010).

Gautier, B. y Vervisch, M.O. (1997): Le manager coach, Dunod, Paris. Traducción de Martin Priego, J.V., (2001): Coaching directivo para el desarrollo profesional de personas y equipos", Oberón, Madrid.

Giménez, F. (2005): “Liderazgo con alma”. Alta dirección 40/239, 11-18.

Hersey, P (1985): Situational Selling: An Approach for increasing Sales Effectiveness, Center for Leadership Studies, Escondido, California.

Hersey, P. y Blanchard, K. (1969): “Life Cycle Theory of Leadership”, Training and Development Journal, mayo.

Jericó, P. (2002): “El líder como coach”. Talento directivo (7), 141-166.

Landsberg, M. (1997): The Tao of Coaching, HarperCollins Publishers, Londres.

Martínez González, J.A. (2011): "Coaching y Liderazgo", Contribuciones a la Economía, Junio. Consultado el 10.10.2011 en http://www.eumed.net/ce/ 2011a/.

Mendoza, R. (2006): "Investigación cualitativa y cuantitativa. Diferencias y limitaciones", Piura, Perú. Consultado el 05.05.2010 en http://www.investiga ci.com/trabajos38/investigacioncualitativa.shtml.

Morse, J.M. (2005), Asuntos críticos en los métodos de investigación cualitativa, Publicaciones Universidad de Alicante.

Muro, P. (2003): El pez que no quiso evolucionar, relatos de la empresa y la vida, Pearson Education, S.A., Madrid.

Piedras, P. (2008): El cuadernillo de Paco, Gestión Directa, Málaga.

Quintana, A y Montgomery, W. (2006): Psicología. Tópicos de actualidad. Lima: UNMSM.

Rodríguez, G., Gil Flores, J. y G쯔 Jiménez, E. (1996), Metodología de la investigación cualitativa, Ediciones Aljibe, Málaga.

Sartre, J.P. (1949): Los caminos de la libertad, Gallimard, Paris.

Yin, R. (1993): “Applications of Case Study Research”, Beverly Hills, Sage. 